\title{
A Cascade Model for Particle Concentration and Enstrophy in Fully Developed Turbulence with Mass Loading Feedback
}

\author{
R. C. Hogan \\ Bay Area Environmental Research Institute; MS 245-3 Moffett Field, CA 94035-1000 * \\ J. N. Cuzzi \\ NASA Ames Research Center; MS 245-3 Moffett Field, CA 94035-100®
}

(Dated: November 4, 2018)

\begin{abstract}
A cascade model is described based on multiplier distributions determined from 3D direct numerical simulations (DNS) of turbulent particle laden flows, which include two-way coupling between the phases at global mass loadings equal to unity. The governing Eulerian equations are solved using psuedo-spectral methods on up to $512^{3}$ computional grid points. DNS results for particle concentration and enstrophy at Taylor microscale Reynolds numbers in the range 34 - 170 were used to directly determine multiplier distributions on spatial scales 3 times the Kolmogorov length scale. The multiplier probability distribution functions (PDFs) are well characterized by the $\beta$ distribution function. The width of the PDFs, which is a measure of intermittency, decreases with increasing mass loading within the local region where the multipliers are measured. The functional form of this dependence is not sensitive to Reynolds numbers in the range considered. A partition correlation probability is included in the cascade model to account for the observed spatial anticorrelation between particle concentration and enstrophy. Joint probability distribution functions of concentration and enstrophy generated using the cascade model are shown to be in excellent agreement with those derived directly from our 3D simulations. Probabilities predicted by the cascade model are presented at Reynolds numbers well beyond what is achievable by direct simulation. These results clearly indicate that particle mass loading significantly reduces the probabilities of high particle concentration and enstrophy relative to those resulting from unloaded runs. Particle mass density appears to reach a limit at around 100 times the gas density. This approach has promise for significant computational savings in certain applications.
\end{abstract}

PACS numbers: 47.61.Jd, 47.27.E-, 47.27.eb

Keywords: Turbulence, Multiphase Flows, Statistical Distributions

\section{INTRODUCTION}

The study of turbulent flows incorporating heavy particles in suspension (particles with finite stopping times) is an important endeavor that has both fundamental and practical relevance to many scientific and engineering problems. Such flows have been investigated mainly in numerical simulations where detailed statistical analysis of the flow fields is possible [1, 2, 3, 4] These simulations, limited to relatively low Taylor microscale Reynolds numbers $R e_{\lambda}(\sim 40)$, demonstrated that particles whose fluid response times are comparable to the lifetime of the smallest turbulent eddies produce a highly nonuniform field with intense regions of concentration. Preliminary indications were that the feedback from such concentrations of particles could locally damp turbulence - however, the role of this "mass loading" effect in determining the statistical distributions of particle density and various fluid scalars has not been thoroughly studied. Experimental investigations of turbulence modification by particles have demonstrated that the degree of turbulence damping increases with particle mass loading and

\footnotetext{
*Electronic address: hogan@cosmic.arc.nasa.gov
}

†Electronic address: jcuzzi@mail.arc.nasa.gov concentration [4].

The phenomenon known as intermittency can be described as intense fluctuations, on small spatial and temporal scales in the turbulent field, that contribute to the exponential tails of probability distribution functions (PDFs) of scalars such as velocity increments and gradients [5, 6, 7], dissipation [8], pressure [9, 10], enstrophy 11, 12] and velocity circulation [13]. Intermittency in the density field of preferentially concentrated particles has also been observed and studied [14, 15].

Although intermittency in turbulence still lacks a complete theoretical understanding, progress has been made with phenomenological models that capture intermittency in a cascade process. Richardson [16] and later Kolmogorov [17] suggested that such models might be used to explain the process of eddy fragmentation initiated by unstable large scale structures in a turbulent fluid. Intermittency in the context of fragmentation though a cascading process has been studied for large-scale gravitating masses [18] and velocity increments in turbulence 19]. Simple cascade models were explored by Meneveau and Sreenivasan [20] and were reviewed by Sreenivasan and Stolovitzky 21] The scale similarity of random fields was explored by Novikov [22, 23], with a focus on the energy dissipation cascade. In Novikov's work, the ratio of dissipation averaged over two spheres, one embedded within the other, served as a measure of enstrophy par- 
titioning between larger and smaller scales. The probability distribution of these ratios, known as multipliers or breakdown coefficients, was shown to relate to multifractal and statistical measures (moments) of the velocity and dissipation fields. A recent review of intermittency in multiplicative cascades stresses that this theory is a kinematic description and its connection with the real dynamics remains unclear 24].

Our previous numerical study of particle concentration in turbulent flows showed that the particle density field is a multifractal on scales comparable to the Kolmogorov length scale [14]. This result suggests that a deeper description of the statistical properties of the particle concentration field, based on multiplier PDFs, may also be possible. Analytical efforts have suggested that dissipation and vorticity in the fluid phase should be locally linked with particle concentration 25]. Numerical work in this regard has demonstrated that preferential concentration is statistically anticorrelated with low vorticity: particles tend to concentrate in regions where enstrophy is relatively weak [26, 27].

In this paper we present a cascade model in the spirit of Novikov [22, 23] that follows the partitioning of positive definite scalars associated with both the fluid and the particles. Multipliers controlling the partitioning of enstrophy and particle density at each step in the cascade are drawn from probability distribution functions (PDFs) which are determined empirically from direct numerical simulations (DNS). Moreover, the multiplier PDFs are dependent on, or conditioned by, the particle mass density or mass loading. The cascade model then generates joint PDFs for particle concentration and enstrophy at arbitrary cascade levels. A partitioning correlation probability is also applied at each cascade level to account for the observed spatial anticorrelation between enstrophy and particle concentration [26, 28].

In Section II we describe the cascade model and its parameters, which are empirically determined from DNS calculations. Details of the DNS equations, and our numerical methods, are discussed in the Appendix. Results are shown in section III, including comparisons of joint PDFs of enstrophy and particle concentration as predicted by the cascade model with those obtained directly from the DNS results. Cascade model PDF predictions at Reynolds numbers well beyond the DNS values are also presented. In section IV, we summarize our results and discuss their implications.

\section{CASCADE MODEL}

A turbulent cascade can be envisioned as an hierarchical breakdown of larger eddies into smaller ones that halts when the fluid viscosity alone can dissipate eddy kinetic energy. Eddies or similar turbulent structures such as vortex tubes are bundles of energy containing vorticity and dissipation. These structures start with a size comparable to the integral scale $\Lambda$ of the flow, and break down in steps to a size comparable to the Kolmogorov scale $\eta$ before being dissipated away by viscosity. The fluid vorticity and dissipation exhibit spatial fluctuations that increase in intensity as the spatial scale decreases. This phenonemon is known as intermittency and has been observed in a variety of processes with strong nonlinear interactions.

In previous numerical and experimental studies, locally averaged intermittent dissipation fields with scale at or near $\eta$ were used to quantify the statistical properties of multiplier distributions [21]. Multipliers are random variables that govern the partitioning of a positive definite scalar as turbulent structures break down along the cascade. In these studies the statistical distribution of multipliers (their PDF) were shown to be invariant over spatial scales that fall within the turbulent inertial range. Multifractal properties of the cascading field are derivable from such multiplier distributions [23], and cascade models based on the iterative application of multipliers to a cascading variable have been shown to mimic intermittency.

While invariant with level in the inertial range of a cascade, multiplier PDFs might depend on local properties of the environment. For instance, Sreenivasan and Stolovitzky [21] showed that the degree of intermittency in dissipation increases with the degree of local strain rate, and constructed multiplier distributions for local energy dissipation conditioned on the local strain rate. The physical mechanism behind this effect is believed to be related to vortex stretching dynamics creating intense bursts of dissipation.

All the multiplier PDFs measured by Sreenivasan and Stolovitzky 21], whether conditioned or unconditioned by local properties, are well characterized by the $\beta$ distribution function,

$$
p(m)=\frac{\Gamma(2 \beta)}{\Gamma(\beta)^{2}} m^{\beta-1}(1-m)^{\beta-1}
$$

where $m$ is the multiplier variable and $\beta$ is a shape controlling parameter. A large $\beta$ produces a narrow, deltafunction-like curve centered at $m=0.5$, whereas $\beta=1$ produces a flat distribution between $m=0$ and 1 . These limits for $\beta$ correspond to uniform and highly intermittent processes respectively. In conditioned multipliers, the value of $\beta$ varies with some local property of the fluid.

Concentration of particles in turbulence is a result of the active dynamics of eddies on all scales. The process depends on the scale of the eddies and the corresponding particle response to those eddies. Intense particle density fluctuations, akin to intermittency, were observed in a previous numerical study where it was also shown that nonuniform particle concentrations have multifractal scaling properties [14]. These results strongly suggest that a phenomenological cascade model based on multipliers may adequately describe the particle density field. Simulations that have included particle feedback on the fluid through the mass loading effect show that damp- 
ing of local turbulence occurs [2, 29]. The latter have shown that vorticity dynamics is affected locally by particle feedback. This interplay between the phases could attenuate vortex stretching and, thereby, diminish local turbulent intermittency. Multiplier distributions conditioned on local mass loading should therefore be an integral part of a realistic fluid-particle cascade model.

\section{A. Two-Phase Cascade model}

Below we describe a two-phase cascade model that incorporates simultaneous multiplier processes for particle concentration $C$ and fluid enstrophy $S$, in addition to a process that models their spatial anticorrelation. The multiplier distributions are conditioned by the local particle concentration, as determined empirically from DNS fields equilibrated to $R e_{\lambda}=34,60,107$, and 170 . The spatial anticorrelation was also quantified from these fields. Local measures of particle concentration $(C)$ and enstrophy $(S)$ used are defined in the Appendix.

A schematic illustration of our two-phase partitioning process is shown in FIG. 1] The cascading vector $(S, C)$ has components representing enstrophy and particle concentration. Initially the components are assigned the value unity and are associated with a common cell having a volume of unity. Each component is partitioned into two parts; $\left(m_{S} S,\left(1-m_{S}\right) S\right)$ and $\left(m_{C} C,\left(1-m_{C}\right) C\right)$, respectively, where $m_{S}, m_{C}$ are multipliers for $S$ and $C$ whose values are between zero and one inclusive and are random members of the corresponding multiplier distributions. The parts are associated with two daughter cells each containing half the volume of the starting cell. In the example shown in FIG. 11 $m_{S}$ and $m_{C}$ are assumed to be greater than 0.5 . The largest parts of $S$ and $C$ are placed in the same daughter cell with probability $\Gamma$ (and in different cells with probability $1-\Gamma$ ). This partitioning process is repeated for each daughter cell down the cascade until the ratio of the daughter cell size to the initial cell size equals a specified cutoff. When this cutoff is set to the ratio of the turbulent lengthscales $\Lambda$ and $\eta$, the cascade corresponds to turbulence characterized by $R e_{\lambda} \sim(\Lambda / \eta)^{2 / 3}[30$.

\section{B. Conditioned Multipliers}

The parameters of the cascade model are empirically derived from the particle density and enstrophy fields $C$ and $S$ as calculated by DNS (see Appendix). The simulation parameters for four DNS runs representing $R e_{\lambda}=$ 36, 60, 104, and 170 are shown in Table I The turbulence kinetic energy $q$, the volume averaged dissipation $\epsilon$, and $\Lambda$ are calculated from the 3-D turbulent energy spectrum $E(k)$ and kinematic viscosity $\nu$,

$$
q=\int_{0}^{\infty} E(k) d k
$$
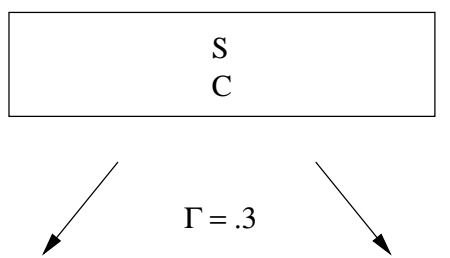

\begin{tabular}{l}
$\mathrm{m}_{\mathrm{S}} * \mathrm{~S}$ \\
$\mathrm{~m}_{\mathrm{C}} * \mathrm{C}$ \\
\hline
\end{tabular}

$$
\left(1-\mathrm{m}_{\mathrm{S}}\right) * \mathrm{~S}
$$$$
\left(1-\mathrm{m}_{\mathrm{C}}\right)^{*} \mathrm{C}
$$
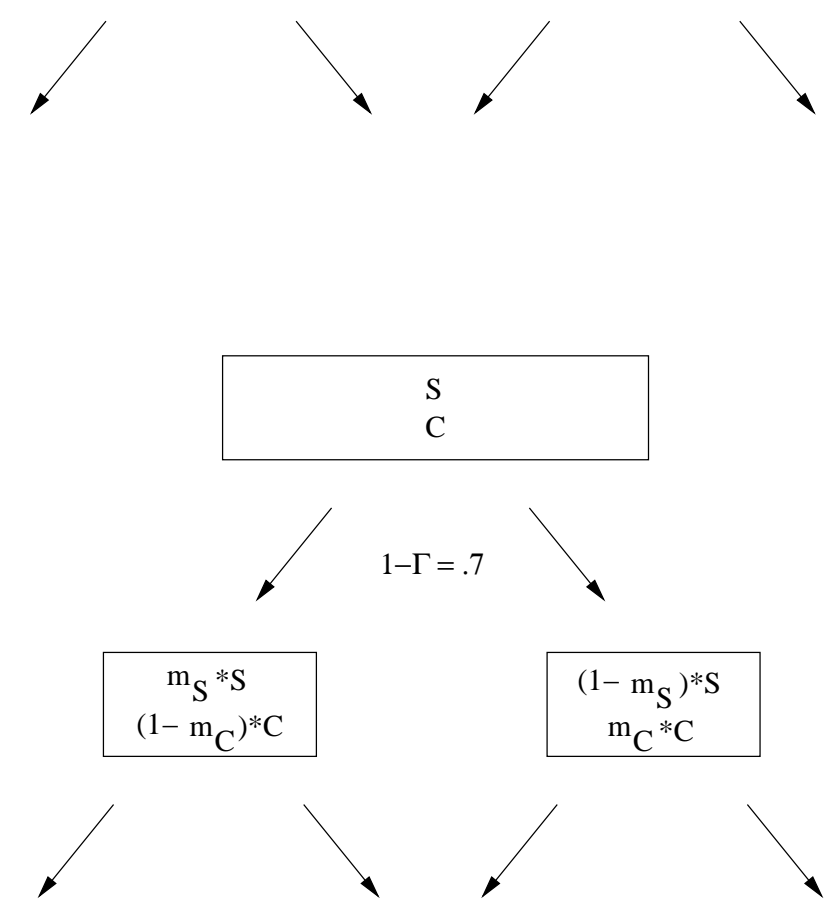

FIG. 1: Figure depicting the breakdown of a parcel of enstrophy $(S)$ and particle concentration $(C)$ into two parcels each with half the volume of the parent. The corresponding multipliers $m_{S}$ and $m_{C}$ are assumed to be greater than 0.5 in this figure. These measures are broken down and distributed between the two parcels in one of two ways - the larger portions are partitioned together with probability $\Gamma=0.3$ (upper figure), or in opposite directions with probability $1-\Gamma=0.7$ (lower figure).

$$
\begin{aligned}
& \epsilon=2 \nu \int_{0}^{\infty} E(k) k^{2} d k \\
& \Lambda=\frac{3 \pi}{4 q} \int_{0}^{\infty} \frac{E(k)}{k} d k
\end{aligned}
$$

where $k$ is wavenumber. $k_{\max }=\frac{\sqrt{2}}{3}$ times the number of computational nodes per side is the maximum effective wavenumber. Thus $k_{\max } \eta>1$ indicates an adequate resolution of the Kolmogorov scale. 


\begin{tabular}{|c|c|c|c|c|}
\hline Parameter & Case I & Case II & Case III & Case IV \\
\hline Nodes/side & 64 & 128 & 256 & 512 \\
$\nu$ & .01 & .003 & .0007 & .0002 \\
$R e_{\lambda}$ & 34 & 60. & 104 & 170 \\
$q$ & 1.5 & .65 & .28 & .14 \\
$\frac{\epsilon}{\nu}$ & 23. & 22.8 & 22.4 & 23 \\
$k_{\max } \eta$ & 1.4 & 1.5 & 1.45 & 1.56 \\
$\frac{\Lambda}{\eta}$ & 14.1 & 23.3 & 45.8 & 86.2 \\
$\Gamma$ & .31 & .29 & .27 & .32 \\
$D$ & .0001 & .00003 & .000007 & .000002 \\
$\nu_{p}$ & .001 & .0003. & .00007 & .00002 \\
\hline
\end{tabular}

TABLE I: Case Parameters for DNS runs. The quantities $D$ and $\nu_{p}$ are defined in the Appendix. Other quantities above are defined in Section II.

The 3-D DNS computational box is uniformly subdivided into spatial cells $3 \eta$ on a side, and the average value of $C$ and $S$ is determined for each cell ( see Appendix ). The cells are divided into groups associated with disjoint ranges of $C$. Each cell is then divided into two parts of equal volume and averages for $C$ and $S$ are determined for each part. The $C$ and $S$ multipliers for each cell are evaluated as the ratio of these averages to the averages in the parent cell. A conditional multiplier distribution $p(m)$ is then determined for each binned value of $C$ from the corresponding set of cell multipliers. Plots of $p(m)$ for three values of $C$ are shown in FIG. 2. The points represent distributions derived from all DNS runs and the solid lines are least squares fits to the $\beta$ distribution function (Eq. 11). For the lower values of $C, R e_{\lambda}$-independence is apparent; only the $R e_{\lambda}=170$ case provided data for the largest $C$ range. The plots clearly indicate that the intermittency in $C$ is reduced (multiplier PDFs narrow) as $C$ is increased. Derived values of $\beta_{C}(C)$ and $\beta_{S}(C)$ are shown as a function of $C$ in FIG. 3. Least squares fits to the functional form $p_{1} \exp \left(p_{2} C^{p_{3}}\right)$ are drawn as solid lines and the best fit parameter values for this function are tabulated in Table II]. Bounding curves (dashed lines) are defined by setting $p_{2}$ and $p_{3}$ to their $2 \sigma$ limits, to establish a plausible range of uncertainty in the predictions.

\begin{tabular}{|c|c|c|c|}
\hline Scalar & $p_{1}$ & $p_{2}$ & $p_{3}$ \\
\hline$C$ & 2.7 & .045 & 1.02 \\
$S$ & 9. & .03 & 1.06 \\
\hline
\end{tabular}

TABLE II: $\beta$ model parameters

It is certainly of interest that such large solid/gas mass loadings as $C=100$ appear in the DNS runs at all, given published reports that particle mass loading significantly dampens turbulent intensity even for mass loadings on the order of unity [1, 4]. These diverse results might be reconciled since the particles we study herein are all far smaller than the Kolmogorov scale and also have only a very small lag velocity relative to the gas. Recall that we force the turbulence, as might be the case if it were
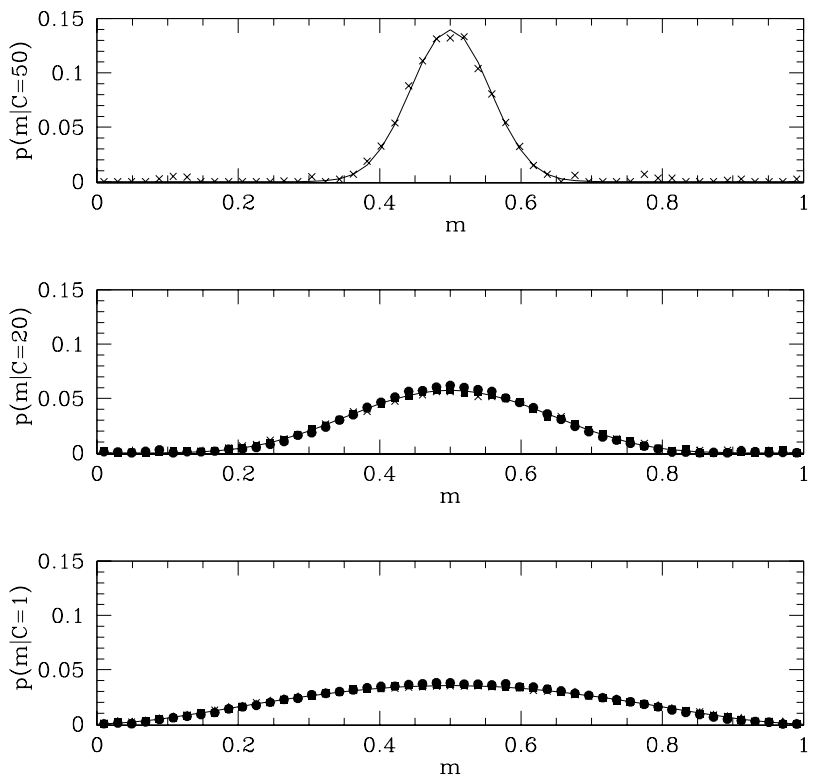

FIG. 2: Empirically determined conditional multiplier distributions $p(m \mid C)$ for particle concentration at three different mass loading values, $C=1,20$ and 50 . The distributions are obtained from bifurcations of cells with a spatial scale equal to $3 \eta$. Results at $R e_{\lambda}=34$ ( square ), 60 (triangle), 107 (circle) and 170 ( cross ) are overlain. Only the simulation with $R e_{\lambda}=170$ provided results for $C=50$. At each mass loading the $p(m)$ at all Reynolds numbers are very well approximated with the $\beta$ distribution function ( solid line ). The distribution widths narrow as the mass loading increases, indicating a decrease in the intermittency.

being constantly forced by energetic sources operating on larger scales than our computational volume. However, FIG. 3 strongly suggests an upper limit for $C(\sim 100)$ for both $\beta_{S}$ and $\beta_{C}$.

The cascade anticorrelation parameter $\Gamma$ was determined by counting the number of parent cells within which the larger partitions of $C$ and $S$ were found to share the same daughter cell. This number divided by the total number of parent cells defines $\Gamma$. The derived $\Gamma$ value is approximately constant across the DNS cases, as indicated in Table [I. Operationally, the $\Gamma$ used in the cascade model was determined by taking a simple average of the $\Gamma$ values in Table 1

Overall, the invariance of $\Gamma$ and the $\beta_{C}(C)$ and $\beta_{S}(C)$ functions across our range of $R e_{\lambda}$ justifies their treatment as level independent parameters in the two-phase cascade model. One caveat remains, which would be of interest to address in future work. While it has been shown that multiplier distributions leading to $\beta_{C}$ and $\beta_{S}$ are level-invariant over a range of scales within an inertial range [21], our simulations were numerically restricted to values of $R e$ in which the inertial range has not yet become fully developed. Our reliance on the smallest available scales of $3 \eta$ to $1.5 \eta$ (those providing the largest available intermittency) might lead to some concern that 


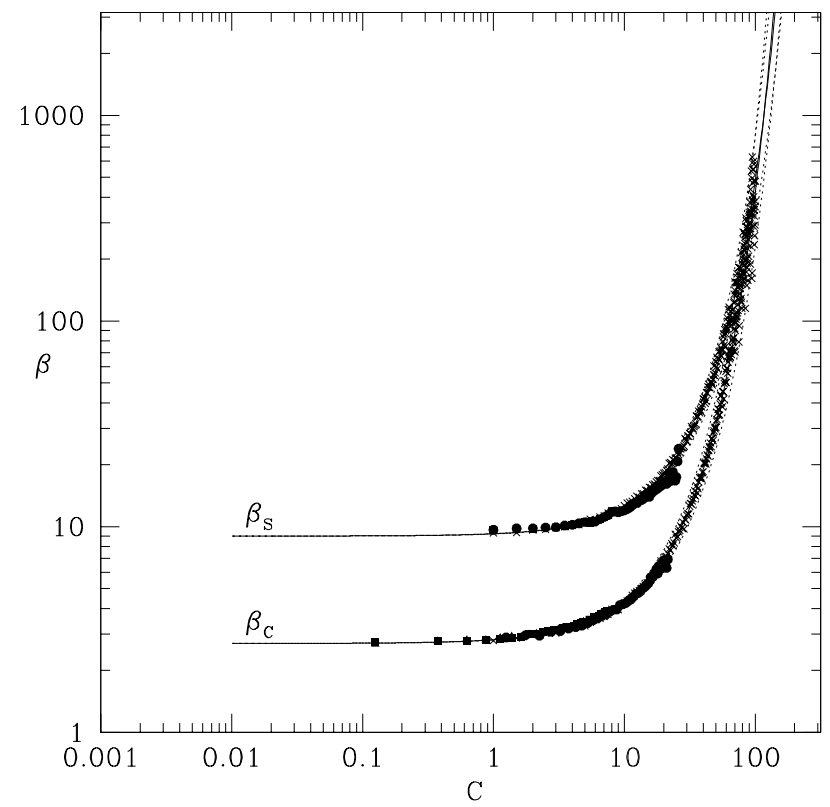

FIG. 3: The $\beta$ parameters as functions of local mass loading $C$ for enstrophy and particle concentration at $3 \eta$. Results for all DNS cases are indicated as described in FIG. 2. A least squares fit of an exponential function to the points over the entire mass loading range is shown ( solid line ). Dashed lines correspond to the upper and lower limits of the function, and are derived using the $2 \sigma$ errors of $p_{2}$ and $p_{3}$.

they were already sampling the dissipation range of our calculations, and thus may not be appropriate for a cascade code. We tested this possibility by calculating multipliers for the next largest level bifurcation ( $6 \eta$ to $3 \eta$ ) for the $R e_{\lambda}=170$ case. The $\beta$ values for those multiplier distributions are slightly larger in value, but consistent with the $C$-dependence shown in FIG. 2 (6 $6 \eta$ scales don't provide good distribution functions beyond $C \sim$ 15). Thus we believe that for the purpose of demonstrating this technique, and for the purpose of estimating the occurrence statistics of $C$ under particle mass loading, our results are satisfactory. For applications requiring quantitatively detailed and/or more accurate $P(S, C)$, it would certainly be of interest to extend the DNS calculations to larger $R e$, at which a true inertial range might be found.

\section{MODEL RESULTS}

The 2D joint probability distribution function or PDF of concentration and enstrophy, a fractional volume measure, was generated from the cascade model and compared with results derived directly from numerical DNS simulations. The basic probability density $P(S, C)$ gives the fractional volume occupied by cells having enstrophy $S$ and concentration $C$, per unit $S$ and $C$; thus the fractional volume having $C$ and $S$ in some range $\Delta S, \Delta C$ is $P(S, C) \Delta S \Delta C$. For quantities varying over orders of magnitude, it is convenient to adopt $\Delta S=S$ and $\Delta C=C$, and we will present the results in the form $P(S, C) S C$.

We started by binning results at spatial scale $3 \eta$, obtained from the semi-final level of a cascade model run, into a uniform logarithmic grid of $S, C$ bins each having width $\Delta(\log S)=\Delta(\log C)=\delta$, with corresponding values of $\Delta S$ and $\Delta C$. The number of $3 \eta$ cells accumulated in each bin was normalized by the total number of such cells in the sample to convert it to a fractional volume $\Delta V(S, C)=P(S, C) \Delta S \Delta C$. Then

$$
\frac{\Delta V(S, C)}{\delta^{2}}=\frac{P(S, C) \Delta S \Delta C}{\Delta(\log S) \Delta(\log C)} \rightarrow P(S, C) S C \quad \text { as } \quad \delta \rightarrow 0 .
$$

In practice of course, the binning ranges $\delta$ are not vanishingly small.

The plots in FIGs. 45 and 7 then, show the PDF as the volume fraction $P(S, C) S C$. Cascade levels 9,12 , 15 , and 18 correspond approximately to the $R e_{\lambda}$ of the four simulation cases shown in Table [I. These levels were determined from the ratio of $\Lambda$ and $\eta$ for each case: level $=3 \log _{2}(\Lambda / \eta)$. The factor 3 accounts for cascade bifurcations of $3 \mathrm{D}$ cells, because it takes three partitionings, along three orthogonal planes, to generate eight subvolumes of linear dimension one-half that of the parent volume. That is, $2^{\text {level }}$ is equal to the number of $\eta$ cells within a 3D volume having linear dimension $\Lambda$ and $\left(2^{\text {level } / 3}\right)^{2 / 3}$ is the corresponding $R e_{\lambda}$. The number of cascade realizations is, in turn, equal to the product of the number of $\Lambda$-size volumes in the computational box and the number of simulation snapshots processed. In general it is difficult to generate DNS results with a ratio of $\Lambda$ and $\eta$ that is an exact power of two. In order to correctly compare DNS simulations with the cascade model it was necessary to interpolate between two cascade generated $P(S, C) S C$ computed at scale ratios (levels) that bracketed the ratios that were actually simulated. In FIG. 4 we compare iso-probability contours of $P(S, C) S C$ predicted by cascade models representing the four DNS cases with the same contours derived directly from the simulated $S$ and $C$ fields. The agreement is very good.

\section{A. Predictions at higher Reynolds number}

The cascade model was used to generate PDFs at deeper levels in order to assess the effect of mass loading on the probabilities of high $C$ and $S$. We generated 256 realizations of a level 24 cascade, 20 realizations of a level 30 cascade, and one realization of a level 36 cascade.

FIG. [5(a) shows the average of 256 realizations of a 24 level cascade, taken to lower probability values. The pronounced crowding of the contours at the top of the figure indicates the effect of particle mass loading on reducing the intermittency of $C$ at high values of $C$. For compar- 


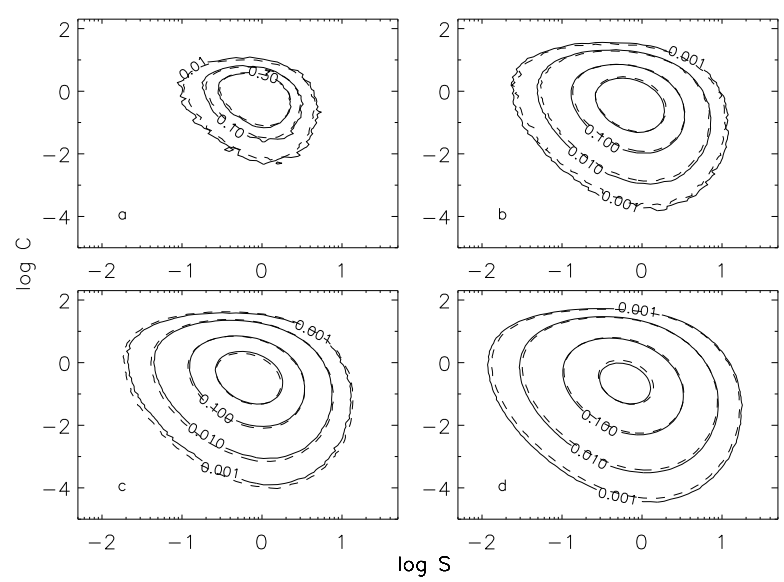

FIG. 4: Comparisons of cascade model predictions of $P(S, C) S C$ with DNS results at $R e_{\lambda}=34$ (a), 60 (b), 107 (c), and 170 (d). Contours indicate probabilities .001, .01, .1 and .3. Dashed contours are cascade model predictions and solid ones are DNS results.

ison, FIG. 5(b) shows a control run of a 24 level cascade with all conditioning turned off. In this control case, the exponential tails characterizing intermittent fluctuations are seen at both low and high $C$.

In order to evaluate the effect of the uncertainties in the extrapolations of the $\beta$ curves for $C$ and $S$ on the PDF, two cascade runs to level 24 were generated using the parameters for the upper and lower dotted curves in FIG. 3. In FIG. 6] we show cross-sections of the PDFs produced by these runs along the $C$ axis through the distribution modes to compare with the same cross-section for a run using the nominal parameters in Table [II Both models diverge from the mean model beyond $C>40$, with the upper (lower) curve corresponding to the outside (inside) $\beta_{C}(C)$ and $\beta_{S}(C)$ bounds in FIG. 3, Figure 6 indicates that the sensitivity of the PDF to the $\beta$ model parameters at the $2 \sigma$ level is only apparent at large $C$, and all models show a sharp dropoff in the probability for $C>$ 100.

A crowding effect similar to the one seen in FIG. 5 (a) is shown in FIG. 7 for iso-probability contours equal to $5 \times 10^{-4}$, for cascade levels $6,12,18,24,30$ and 36 .

Figures $8(a)$ and $8(b)$ compare $1 \mathrm{D}$ cuts through the modes of the PDFs for cascades of 18 - 36 levels, indicating that going to deeper levels (higher $R e_{\lambda}$ ) results in larger intermittency at the low- $C$ end (as expected), retaining the exponential tail characteristic of intermittent processes, but the highest particle concentration end of the distribution is extended more slowly. Certainly at the order of magnitude level, a particle mass loading ratio of 100 times the gas density appears to be as high as preferential concentration can produce. This result could be inferred directly from inspection of the conditioned $\beta$ distributions of FIG. 3 ,
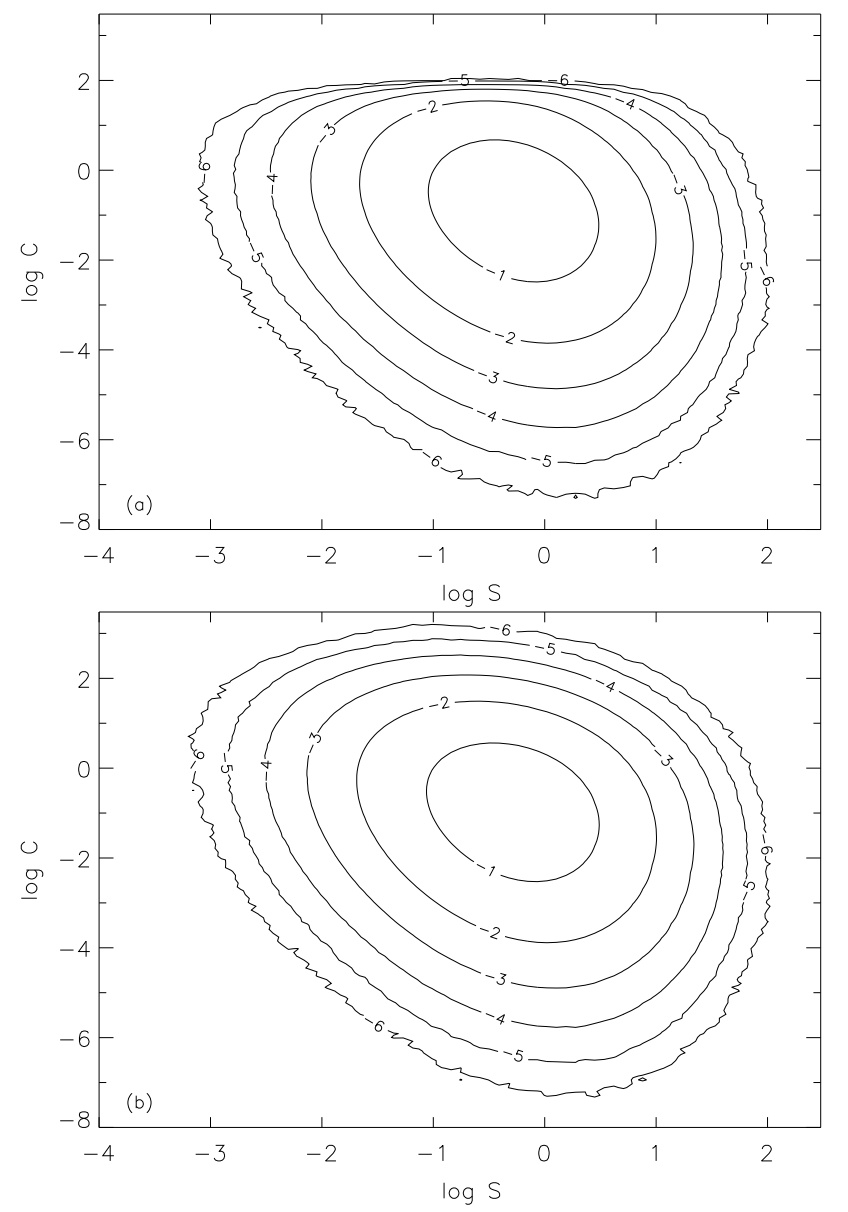

FIG. 5: (a) Cascade model predictions for a 24 level case, taken to lower probability levels, using 256 realizations of the cascade. Contours are labeled by $\log (P(S, C) S C)$. Note the crowding of contours at high $C$ values, indicating the high- $C$ limit of the process under conditions of mass loading.(b) A control cascade to level 24, as in FIG. [5(a), with conditioning turned off. The difference between (a) and (b) clearly shows the "choking" effects of particle mass loading on intermittency in $C$.

\section{SUMMARY}

A two-phase cascade model for enstrophy and particle concentration in 3-D, isotropic, fully developed turbulence with particle loading feedback has been developed and tested. Multiplier distributions for enstrophy and particle concentration were empirically determined from direct numerical simulation fields at Taylor scale Reynolds numbers between 34 and 170 . These simulations included 'two-way' coupling between the phases at global particle/gas mass loadings equal to unity. The shape of all multiplier distributions is well characterized by the $\beta$ distribution function, with a value of $\beta$ that depends systematically on the local degree of mass loading. The values of $\beta$ increase monotonically with mass loading and begin to rapidly increase at mass loadings 


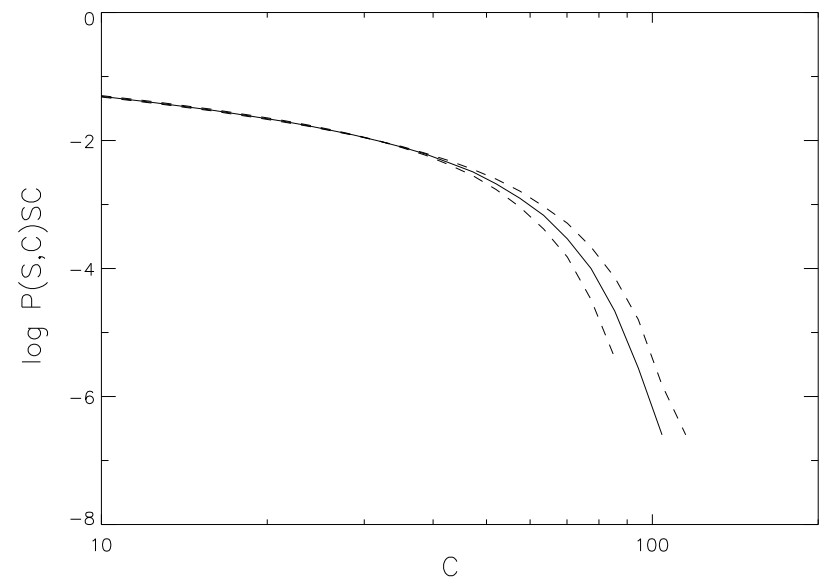

FIG. 6: 1D cuts through the mode of the PDF of FIG. 51(a) parallel to the $C$ axis, showing the effects of uncertainty in the conditioning curve $\beta_{C}(C)$. The solid curve is the nominal model and the dashed curves are obtained by allowing the parameters $p_{2}$ and $p_{3}$ to take their $2 \sigma$ extreme values.

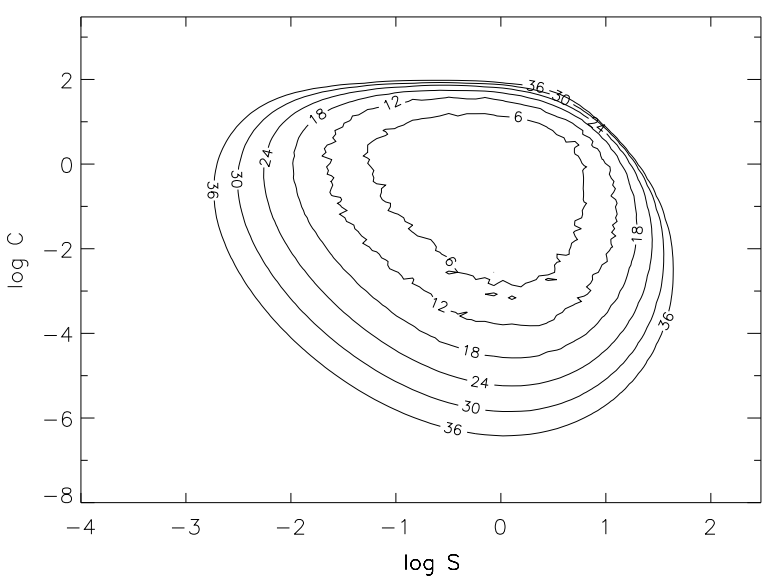

FIG. 7: Cascade model predictions for $P(S, C) S C=5 \times 10^{-4}$ for levels $6,12,18,24,30$, and 36 . Contour labels indicate the cascade levels.

greater than 100 .

The $C$-dependent multiplier distributions were used as input to a cascade model that simulates the breakdown, or cascade, of enstrophy $S$ and particle concentration $C$ from large to small spatial scales. The spatial anticorrelation between enstrophy and particle concentration was empirically determined from 3D DNS models and shown to be constant with $R e_{\lambda}$. This constant was used as a correlation probability governing the relative spatial distribution of $S$ and $C$ at each bifurcation step in the cascade model.

The cascade model we have developed clearly reproduces the statistical distributions and spatial correlations observed in our DNS calculations. The cascade parameter values we have derived appear to be universal within
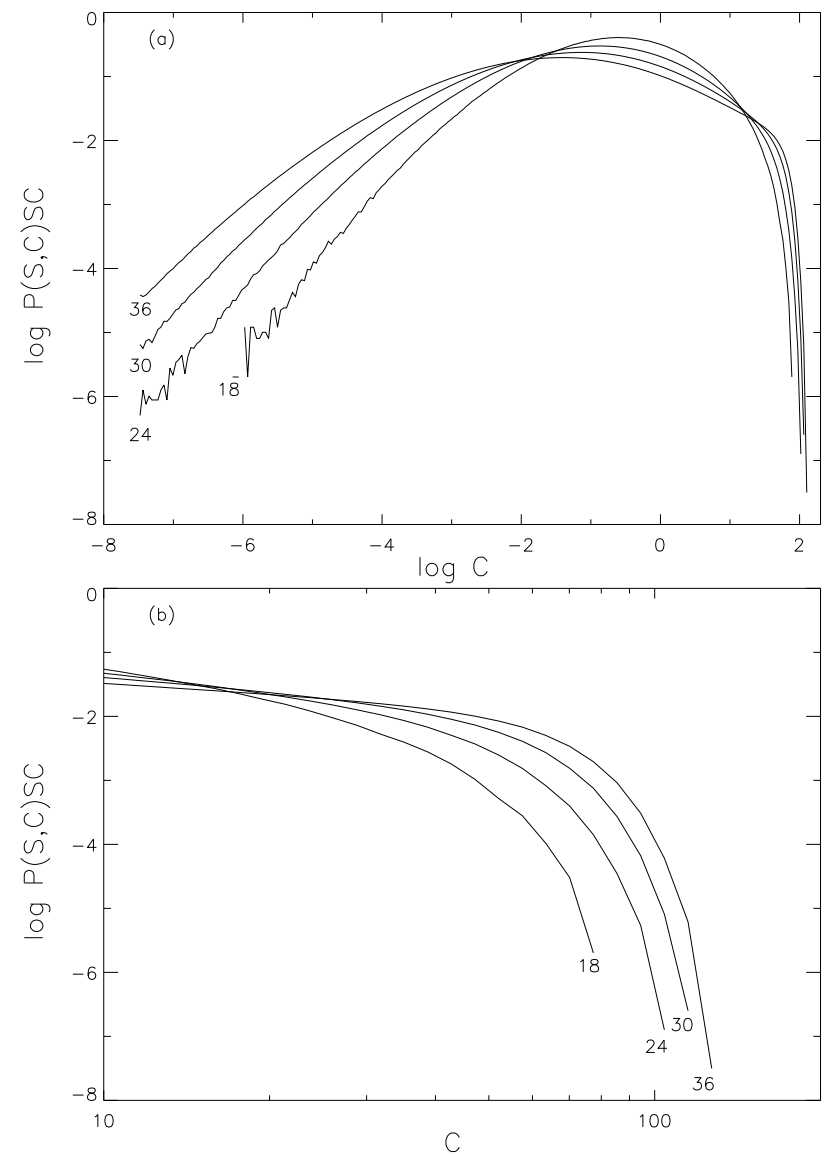

FIG. 8: (a) 1D global cuts through the cascade model PDFs $P(S, C) S C$ for runs with $18,24,30$, and 36 levels. (b) closeup of $1-\mathrm{D}$ cuts through high- $C$ regime.

the range of $R e_{\lambda}$ of our simulations. We thus speculate that they can be used to predict approximate joint probabilities of enstrophy and particle concentration at higher Reynolds numbers, at great savings in computer time. For example, a typical DNS run to $R e_{\lambda}=170$ takes about $170 \mathrm{cpu}$ hours on an Origins 3000 machine, while a cascade model to an equivalent level takes $0.1 \mathrm{cpu}$ hours.

We have presented joint probabilites of $S$ and $C$ derived from cascade runs up to level 36 . The contours shown in FIG. 5(a) and FIG. 6 clearly show the effects of particle mass loading on the probability distribution functions of $C$ in the regimes where $C$ is large. It appears that particle mass loadings greater than 100 are rare in turbulent flows.

The properties of the cascade rest on the physics of our DNS simulations, and we speculate that two separate effects are involved. First, particle mass loading dampens fluid motions of all types, decreasing vorticity stretching and all other forms of ongoing eddy bifurcation which are needed to produce intermittency. Second, as a byproduct of this, particle mass loading may alter the Kolmogorov timescale locally and shift the most effectively concen- 
trated particle Stokes number $S t$ to a larger value than that characterizing particles already lying in the local volume, reducing the probability of preferentially concentrating the local particles any further.

Caveats and Future Work:

As described in section II, our multiplier distributions were taken from the most numerous cells, with the largest intermittency, which are at the smallest scales possible (furthest from the forcing scale). At Reynolds numbers accessible to DNS, a true inertial range is only beginning to appear, and while, sampling at the smallest spatial scales possible, we are as closely approaching the asymptotic values within the true inertial range as possible, where level-independence has been demonstrated in the past [21], it is possible that our values are subject to inaccuracy by virtue of being sampled too close to the dissipation scale. Any such inaccuracy will affect our cascade results quantitatively but not qualitatively. As computer power increases, it would be a sensible thing to continue experiments like these at higher $R e_{\lambda}$.

A more general model that treats enstrophy and strain as independent cascading scalars might allow for a higherfidelity particle concentration cascade, since $C$ is known to be linked to the difference between these two scalars [25] (the so-called second invariant tensor $I I$ ). However, such an effort would introduce further complexity of its own, as $I I$ is no longer positive definite. We consider the development of such a model a suitable task for future work.

\section{APPENDIX}

We used an Eulerian scheme developed by Dr. Alan Wray to solve the coupled set of fluid/particle equations used in this study. This was done to maximize the computational efficiency of the calculations and, more importantly, to accurately evaluate multipliers over the wide range of particle concentrations and enstrophies expected. In this study the effects of particle collisions and external forces on the particles (e.g., gravity) are not considered. The turbulence is spectrally forced at $k=\sqrt{14}$ such that moments of the Fourier coefficients of the force satisfy isotropy up to the fourth order. The instantaneous Navier-Stokes equations describing the conservation of mass and momentum for an incompressible fluid are

$$
\begin{gathered}
\nabla \cdot \mathbf{U}=0 \\
\frac{\partial \mathbf{U}}{\partial t}+(\mathbf{U} \cdot \nabla) \mathbf{U}=-\frac{\nabla P}{\rho_{f}}+\nu \nabla^{\mathbf{2}} \mathbf{U}-\alpha \frac{\rho_{p}}{\rho_{f}}(\mathbf{U}-\mathbf{V})
\end{gathered}
$$

where $\mathbf{U}$ is fluid velocity, $\mathbf{V}$ is particle velocity, $\rho_{f}$ and $\rho_{p}$ are the fluid and particle mass densities, $\nu$ is fluid viscosity, $P$ is pressure, and $\alpha$ is the inverse of the particle gas drag stopping time $\tau_{p}$.
The compressible equations for the particles are

$$
\frac{\partial \rho_{p}}{\partial t}+\nabla\left(\rho_{p} \mathbf{V}\right)=D \nabla^{2} \rho_{\mathbf{p}}
$$

$$
\frac{\partial\left(\rho_{p} \mathbf{V}\right)}{\partial t}+\nabla\left(\rho_{p} \mathbf{V} \mathbf{V}\right)=\nu_{p} \nabla^{\mathbf{2}}\left(\rho_{p} \mathbf{V}\right)+\alpha \rho_{p}(\mathbf{U}-\mathbf{V})
$$

where $\nu_{p}$ is a "particle viscosity", and $D$ is a "particle diffusivity". The particle diffusivity and viscosity terms numerically smooth out particle mass and momentum, alleviating the formation of steep gradients of $\rho_{p}$ that can lead to numerical instabilities eg. 31.

The right hand sides of Eqs. A.2 and A.4 contain phase coupling terms which are linearly dependent on $(\mathbf{U}-\mathbf{V})$. The linear form of the coupling follows from the assumptions that the particle size is much less than $\eta$, and that the material density of the particles is much greater than $\rho_{f}$ [2]. Additional contributions to the particle-gas couplings involving pressure, viscous and Basset forces [29] have not been added since they are expected to be weak in our size regime of interest. The particle field is introduced with a constant mass density and an initial velocity given by the local gas velocity in a field of statistically stationary turbulence. All runs are continued until the particle statistics (RMS of conentration distribution) have equilibrated.

The particle Stokes number $S t$ is defined relative to the Kolmogorov time scale $\tau_{\eta}$ as $S t=\tau_{p} / \tau_{\eta}$, and $\Phi=$ $M_{p} / M_{f}$ is the global mass loading, where $M_{p}$ and $M_{f}$ are the total mass of particles and fluid respectively. In this study $\rho_{f}, S t$, and $\Phi$ are set to unity, $D / \nu=0.01$, and $\nu_{p} / \nu=0.1$. Explicitly setting $S t=1$ guarantees that the particles are preferentially concentrated. When $\Phi$ is unity, $\rho_{p}$ is a surrogate for the local mass loading or local concentration factor $C$. The values of $\nu_{p}$ and $D$ minimize the diluting effects of numerical particle diffusion while preventing numerical blowups; their values were determined from a set of DNS runs in which their values were decreased systematically until numerical instabilities set in.

Eqs. A.1 - A.4 are solved using psuedo-spectral methods commonly used to solve Naviers-Stokes equations for a turbulent fluid. The Fast Fourier Transform (FFT) algorithm is used to efficiently evaluate the dynamical variables $\mathbf{U}, \mathbf{V}$ and $\rho_{p}$ on a $3 \mathrm{D}$ uniform grid of computional nodes with periodic boundary conditions. The computational algorithm is parallelized using MPI and is written in Fortran 90. All runs for this study were executed on SGI Origins supercomputers with up to 1024 processors.

Enstrophy is defined as

$$
S=\frac{1}{2} \sum_{i j}\left(\partial_{i} \mathbf{U}_{\mathbf{j}}-\partial_{j} \mathbf{U}_{\mathbf{i}}\right)^{2}
$$

where $i, j$ are summed over the three coordinate dimensions of $\mathbf{U}$. 
The local spatial average of a scalar over a sample volume is estimated as,

$$
\frac{1}{n d v} \sum_{i}^{n} F_{i} d v
$$

where $F_{i}$ is the scalar's value on computational node $i$ centered within a cube of volume $d v$ and the sum is over all $n$ nodes covering the sample volume. We normalized this average by the global average value to get a quantity that measures the scalar's local value relative to its mean. In this paper $C$ and $S$ will denote normalized spatial averages of particle concentration and enstrophy over cubes $3 \eta$ on a side.

\section{ACKNOWLEDGMENTS}

We are very grateful to Dr. Alan Wray for providing the 3-D code and for useful comments on its use. We thank Robert Last for parallelizing the cascade code on the SGI Origins 3000. We also would like to thank the consultants and support staff at the NAS facility for providing invaluable assistance, and the Science Mission Directorate of NASA for generous grants of computer time. We thank Prof. K. Sreenivasan for several helpful conversations in the preliminary stages of this project and the internal reviewers Drs. Alan Wray and Denis Richard for their suggestions for improving the manuscript. This research has been made possible by a grant from NASA's Planetary Geology and Geophysics program.
[1] K. D. Squires and J. K. Eaton, Phys. Fluids A 2, 1191 (1990).

[2] K. D. Squires and J. K. Eaton, Tech. Rep. MD-55, Stanford University (1990).

[3] K. D. Squires and J. K. Eaton, Phys. Fluids. A 3, 1159 (1990).

[4] J. D. Kulick, J. R. Fessler, and J. K. Eaton, J. Fluid Mech. 227, 109 (1994).

[5] B. Castaing, Y. Gagne, and E. J. Hopfinger, Physica D 46, 177 (1990).

[6] S. P. G. Dinavahi, K. S. Breuer, and L. Sirovich, Phys. Fluids 7, 1122 (1995).

[7] P. Kailasnath, K. R. Sreenivasan, and G. Stolovitzky, Phys. Rev. Lett. 68, 2766 (1992).

[8] A. Vincent and M. Meneguzzi, J. Fluid Mech. 225, 1 (1991).

[9] A. Pumir, Phys. Fluids 6, 2071 (1994).

[10] E. Lamballais, M. Lesieur, and O. Métais, Phys. Rev. E 56, 6761 (1997).

[11] J. Jiménez, A. A. Wray, P. G. Saffman, and R. S. Rogallo, J. Fluid Mech. 255, 65 (1993).

[12] G. He, S. Chen, R. H. Kraichnan, R. Zhang, and Y. Zhou, Phys. Rev. Lett. 81, 4636 (1998).

[13] N. Cao, S. Chen, and K. R. Sreenivasan, Phys. Rev. Lett. 76, 616 (1996).

[14] R. C. Hogan, J. N. Cuzzi, and A. R. Dobrovolskis, Phys. Rev. E 60, 1674 (1999).

[15] E. Balkovsky, G. Falkovich, and A. Fouxon, Phys. Rev. Lett. 86, 2790 (2001).
[16] L. F. Richardson, Weather Prediction by Numerical Process. (Cambridge University Press, Cambridge U.K., 1922).

[17] A. N. Komolgorov, J. Fluid Mech. 13, 82 (1962).

[18] T. Chiueh, Chin. J. Phys. 32, 319 (1994).

[19] M. Gorokhovski, Tech. Rep., Center for Turbulence Research, Annual Research Briefs (2003).

[20] C. Meneveau and K. R. Sreenivasan, Phys. Rev. Lett. 59, 1424 (1987).

[21] K. R. Sreenivasan and G. Stolovitzky, J. Fluid Mech. 379, 105 (1995).

[22] E. A. Novikov, Phys. Fluids A 2, 814 (1990).

[23] E. A. Novikov, Phys. Rev. E 50, R3303 (1994).

[24] J. Jiménez, J. Fluid Mech. 409, 99 (2000).

[25] M. R. Maxey, Phys. Fluids 30, 1915 (1987).

[26] K. D. Squires and J. K. Eaton, J. Fluid Mech. 226, 1 (1991).

[27] A. M. Ahmed and S. Elghobashi, Phys. Fluids 13, 3346 (2001).

[28] J. K. Eaton and J. R. Fessler, Int. J. Multiphase Flow 20, Suppl., 169 (1994).

[29] S. Elghobashi and G. C. Truesdell, Phys. Fluids A 5, 1790 (1993).

[30] U. Frisch, Turbulence (Cambridge University Press, Cambridge, U.K., 1995), chap. 8.

[31] A. Johansen, A. C. Anderson, and A. Brandenburg, Astron. Astrophys. (2004). 